\title{
Strategic Research on the Molecular Diagnostics Industry in China
}

\author{
Xie Lan, Liu Ran, Feng Juan, Xing Wanli, Cheng Jing \\ Medical Systems Biology Research Center, School of Medicine, Tsinghua University, Beijing 100084, China
}

\begin{abstract}
Molecular diagnostics is an important part of personalized medicine and has become the main driving force of the in vitro diagnostics industry. In recent years, China's molecular diagnostics industry has been growing rapidly, despite its small size. Based on literature research, expert interviews, and field investigation, we performed an intensive market analysis of the current situation of the domestic molecular diagnostics industry. The aim of this study is to explore the characteristics of the domestic molecular diagnostics industry; identify opportunities and challenges in innovation, industrial transformation, and related policies and regulations; and propose approaches to overcome existing constraints under the current administrative system. This analysis is of great significance for promoting the development of China's molecular diagnostics industry.
\end{abstract}

Keywords: molecular diagnostics; in vitro diagnostics; market analysis; policies and regulations; biochip

\section{Introduction to the molecular diagnostics industry and significance of strategic research on the development of the molecular diagnostics industry}

\subsection{Introduction to molecular diagnostics techniques}

In November 1999, the American Society for Investigative Pathology and Association for Molecular Pathology jointly published The Journal of Molecular Diagnostics, marking the advent of the molecular diagnostics filed [1].

Molecular diagnostics is based on molecular biology theory, which uses molecular biological techniques and methods to study the structures or expression changes of human endogenous or exogenous biological macromolecules, as well as to provide information and a foundation for the prevention, diagnosis, and treatment of diseases [1]. Reviewing the development of molecular diagnostics during the past 20 years, one can divide the field into three stages:
(1) Nucleic acid hybridization was utilized to diagnose genetic diseases.

(2) Advancements in polymerase chain reaction (PCR) technology promoted the rapid development of molecular diagnostics, particularly the popularization and application of quantitative PCR and real-time PCR.

(3) Numerous high-throughput detection technologies, represented by biochip technology, were developed, breaking through the limitations of traditional detection methods. These technologies are characterized by high sample processing capacity, high-throughput, high-integration, and automation and have become an emerging force in molecular diagnostics [1]. Biochip technology includes microarray and microfluidic chips and is applied to analyze various biomolecules, including genes, proteins, cells, and tissues. This technology shows potential for different applications and possesses commercial value.

Thus far, DNA hybridization and PCR are considered the most basic and common methods in molecular diagnostics. However, technologies such as microarray chips and

Received date: December 18, 2016; Revised date: March 22, 2017

Corresponding author: Cheng Jing, Chinese Academy of Engineering, Academician; Medical Systems Biology Research Center, School of Medicine, Tsinghua University, Professor. Major research fields include biotechnology researches in basic science, clinical medicine and health management. E-mail: jcheng@tsinghua. edu.cn

Funding program: CAE Advisory Project “Strategic Research on the Molecular Diagnostics Industry in China” (2014-XZ-05)

Chinese version: Strategic Study of CAE 2017, 19 (2): 029-036

Cited item: Xie Lan et al. Strategic Research on the Molecular Diagnostics Industry in China. Strategic Study of CAE, https://doi.org/10.15302/J-SSCAE-2017.02.005 
second-generation sequencing with microfluidic chips have been rapidly developed and extensively investigated.

\subsection{Significance of strategic research on the development of the molecular diagnostics industry}

Modern medicine has been undergoing a shift from standardized medicine to personalized medicine. Personalized medicine is a new medical model that considers disease early warning, diagnosis, prognostic evaluation, and medication guidance (mainly at the genetic level) to achieve accurate diagnosis and effective treatment. Molecular diagnostics is an important component of personalized medicine, and the continuous development of molecular diagnostics has advanced from disease diagnosis and treatment to whole life health monitoring, focusing on the prevention of diseases and improvement in the overall population health status. This field has shown a remarkable social economic importance. In 2015, President Barack Obama, in his State of the Union address, announced the launch of a precision medicine program amounting to 215 million USD. Precision medicine has been widely explored based on its core idea, i.e., to fully consider individual differences in terms of genes, environment, and lifestyle and improve the effectiveness of disease prevention. Thus, molecular diagnostics is an indispensable part of precision medicine.

In terms of application maturity and market acceptance, molecular diagnostics applications include: (1) rapid and sensitive diagnosis of infectious diseases and drug resistance (including blood screening); (2) genetic disease detection used for reproductive health (including chromosomal disease detection); (3) early tumor diagnosis, molecular typing, and targeted therapy; and (4) pharmacogenomics.

\section{Research progress on the global molecular diagnostics industry}

\subsection{Global molecular diagnostics industry is undergoing rapid development}

In vitro diagnostics (IVD) refers to the use of medical instruments and reagents to assess specimens of human blood, urine, feces, tissues, and other body fluids to identify diseases and infections. According to a report on IVD by Allied Market Research, the global IVD market was worth 53.3 billion USD in 2013 and is expected to reach 58.8 billion USD in 2018 and 74.7 billion USD in 2020 [2]. The United States is a global IVD innovation center with the largest market, showing a 3\%-5\% annual compound growth rate (CAGR) per year, and its market is predicted to reach 30.1 billion USD in 2020. Asia has the fastest growth rate with a CAGR of $7.49 \%$ [2].

Among the many market segments of the IVD industry, molecular diagnostics is the most prominent and considered a major growth driver. The proportion of molecular diagnostics to IVD has increased from $2 \%$ in 1995 to $11 \%$ in 2012. In 1995, only 100 companies were engaged in molecular diagnostics. However, with the rapid global development of molecular diagnostics methods, this number has increased to more than 500 in 2010 [3]. Some of the most well-known companies include technology providers such as Thermo Electron Corporation (known as Thermo Fisher Scientific), Agilent Technologies, Illumina, GE, and Siemens AG, as well as drug manufacturers such as Roche, Bayer Group, and Abbott Pharmaceutical Co., Ltd. Molecular diagnostics also has a high degree of market centralization.

\subsection{Development rate of the molecular diagnostics industry in China is higher than that in other countries, with broad market prospects}

Molecular diagnostics in China initially emerged in the 1960s and 1970s. Various technologies, such as radionuclide labeling based on nucleic acid probes, dot blotting, Southern blotting, and restriction fragment length polymorphism linkage analysis, were developed in the $1980 \mathrm{~s}$. Since the popularization and application of PCR technology in the 1990s, molecular diagnostics techniques have been rapidly established in China and applied in clinical laboratories.

In 2013, the global IVD market reached 53.3 billion USD, whereas the IVD market in China was approximately 20 billion CNY [4]. In the sub-sectors of the IVD industry in China, biochemical diagnostic technology has entered a mature period and become nationalized with an annual growth rate of less than $10 \%$. Immunological diagnosis is rapidly developing with chemiluminescence methods, and the industrial growth rate of this technique is between $15 \%$ and $20 \%$. Because of the advantages of convenience and low-cost, point-of-care testing (POCT) has been growing at a rate of approximately $24 \%$. Molecular diagnostics is considered the fastest growing sub-industry in the IVD industry, with an annual growth rate of approximately $30 \%$ (Fig. 1) [5], which is 2-fold higher than the global average annual growth rate.

Despite its small size, China's molecular diagnostics market is growing rapidly. Its market size increased from 1.65 billion CNY in 2010 to approximately 4.59 billion CNY in 2014, with a CAGR of $29.1 \%$, and its share of the IVD market increased from $11 \%$ in 2010 to $15 \%$ in 2014 . The growth rate of the molecular diagnostics market is higher than the overall growth rate of IVD [5].

After more than 20 years of development, the molecular diagnostics industry in China has shown great potential for industrialization. More than 150 enterprises and other institutions participate in the research and development of products related to molecular diagnostics, including CapitalBio Co., Ltd., Daan Gene Co., Ltd., United Gene Group, and the Beijing Genomics Institute. 


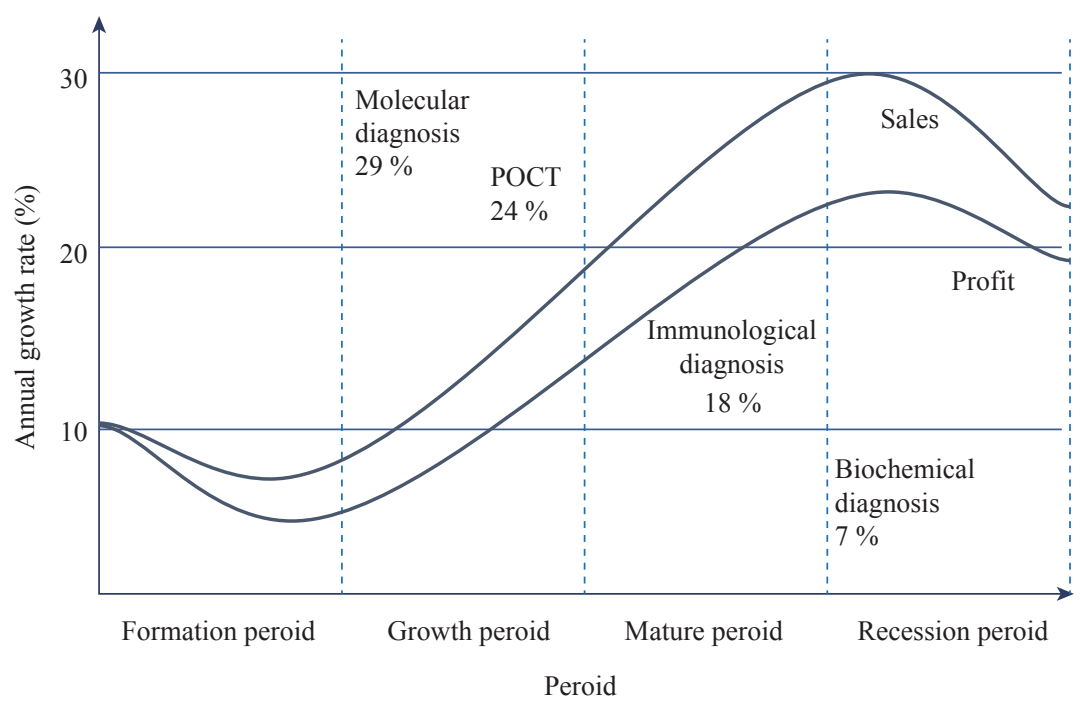

Fig. 1. Development of molecular diagnostics industry in China [5].

Similar to the global molecular diagnostics market, PCR is an advanced molecular diagnostics technology in China and accounts for the largest marker share (approximately 40\%) [5]. Gene chip technology and microfluidic chip-based secondgeneration sequencing technology also have good prospects.

\subsection{New IVD technologies are successively emerging and developing rapidly}

Although PCR remains a market-accepted and relatively mature technology, a series of new technologies has emerged in the field of IVD and shown potential for future clinical applications.

\subsubsection{Continuous innovation in PCR technology}

After years of development, many technologies such as multiplex PCR, nested PCR, reverse transcription PCR (RT-PCR), and real-time fluorescence quantitative PCR have been derived from conventional PCR. With RT-PCR, molecular diagnostics can be used to extend beyond the DNA level and detect defective genes at the RNA level. Thus, pathogens such as viruses that use RNA as their genetic material can be conveniently detected. The most widely used fluorescent quantitative PCR technology adds a specific fluorescent probe to nucleic acid amplification. Thus, amplification can be monitored in real time and quantitative detection can be performed.

Digital PCR is a tool for absolute quantification of DNA based on single-molecule PCR. A large amount of diluted DNA is dispersed into micro-reactors via microfluidic techniques. The number of DNA templates per reactor is less than or equal to 1 [6]. After the PCR is complete, the fluorescence signal in each micro-reaction well is measured. The wells containing the target sequence template fluoresce, whereas the wells without target sequence template produce no signal. Based on these observations, one can calculate the concentration of a nucleic acid to be tested in the original solution. A droplet digital PCR instrument developed by QuantaLife, Inc., which was acquired by Bio-Rad in 2011, has been used in many fields, such as pathogen detection and single-cell gene expression detection.

2.3.2 Advancements in non-nucleic acid detection technology

In addition to the direct detection of nucleic acids, the detection of non-nucleic acid biomolecules (e.g., proteins) is an important aspect of molecular diagnostics. Protein chips remarkably improve the throughput of protein detection by methodically fixing proteins on glass and other solid-phase media and then using a fluorescently labeled antibody to react with the targets. Fluorescence reveals the corresponding protein levels. This method can be used for high-throughput protein detection.

\subsubsection{Advancements in sequencing technology}

Sequencing technologies are unique means by which nucleic acid sequences can be directly obtained, and such technologies are an important component of molecular diagnostics techniques. The termination method developed by Sanger in 1977 is the most classic first-generation sequencing technique and remains the most commonly used method to obtain nucleic acid sequence information. In 2005, 454 Life Sciences, Inc. (acquired by Roche) launched a pyrosequencing-based high-throughput genome sequencing system that pioneered second-generation sequencing. In contrast to first-generation sequencing, second-generation sequencing involves simultaneous synthesis and sequencing. In this technique, a DNA library is formed by DNA fragmentation and expanded after a fragment is cross-linked to a carrier. This library is then simultaneously sequenced and synthesized on the carrier. Second-generation sequencing has rapidly expanded the throughput of gene sequencing, enabling simultaneous detection of large amounts of DNA. Therefore, this technique is also known as deep sequencing or next-generation sequencing. 
After more than a decade of development, second-generation sequencing has become advanced and commercialized. Common platforms include Roche 454, Illumina Solexa, ABI SOLID, and Life Ion Torrent, but the Roche 454 and ABI SOLID systems have been phased out. Breakthroughs have been made in the metagenomics identification of pathogenic microorganisms, analysis of fetal DNA in maternal blood for prenatal diagnosis, and pharmacogenomics for personalized medicine. However, the development of second-generation sequencing presents new requirements compatible with developments in the bioinformatics field.

Because second-generation sequencing is continuously under development, third-generation sequencing technology characterized by single-molecule DNA sequencing has emerged. In 2008, Helico BioScience first reported a single-molecule sequencing technology based on total internal reflection microscopy (TIRM) [7] and successfully launched the third-generation sequencers PacBio RS II and PacBio Sequel. Pacific Biosciences further developed single-molecule real-time (SMRT) sequencing technology, and Oxford Nanopore Technologies developed nanopore sequencing technology. These technologies may also be useful for molecular diagnostics in the future.

\subsubsection{Continuous POCT development}

POCT and molecular diagnostics are the two fastest growing fields of IVD. POCT products have three characteristics: a short detection time, flexible detection locations, and easy operation. The development goal of a POCT platform is to fully integrate multi-step detection into one system so that users only need to add a sample, and the system can finish the remaining steps fully automatically, thus achieving "sample in, product out." The terminal data generated by POCT can be transmitted to medical institutions and provide remote services for customers.

\subsubsection{Gradual maturation of molecular imaging technology}

Molecular diagnostics refers to "in vitro diagnostics," i.e., IVD is conducted after samples are collected from test subjects. With the development of medical imaging technology, "in vivo" molecular diagnostics or in vivo molecular imaging has become available. In molecular imaging, cellular functions can be visualized and molecular processes within living organisms can be monitored noninvasively. This is essential for the early diagnosis and typing of tumors, drug target detection, and prognostic evaluation of many diseases, particularly tumors. Molecular imaging involves molecular probes, systematic measurement, data analysis, and processing [8]. This technology may be further improved for multi-modal molecular imaging. Su et al. [9] targeted epidermal growth factor receptor (EGFR) by using a ${ }^{64} \mathrm{Cu}$-labeled heterogeneous nanocomposite structure material with gold-iron oxide and visualized EGFR at different levels by PER, MRI, and optical tri-modal imaging, which can sensitively detect EGFR expression. Although molecular imaging has been applied in the field of cancer diagnosis and treatment, its clinical application has been unsuccessful because of the high cancer heterogeneity, complex microenvironments, and special anatomical features. Therefore, molecular imaging requires improvement before it can be widely applied.

\subsection{National policy support and market environment}

In China, the supervision environment of industries related to genomics has experienced a lax period (before 2014), stagnation period (February 2014), partially released period (from June 2014 to December 2014), and encouraging period (2015 to now). IVD is an important development area in the 13th Fiveyear Plan. Supervision departments are gradually supporting the development of this industry, creating a rare opportunity for the IVD industry.

As pressure on the control of medical insurance fee increases, hospital revenue sources are gradually shifted from medicines to medical services. The status of IVD, including molecular diagnostics, is slowly rising [5]. On January 5, 2016, the State Council issued the Decision on Implementing the Universal Two-child Policy and Reforming and Improving the Management of Family Planning Services. In the field of non-invasive prenatal screening alone, the two-child policy leads to the extra birth of millions of newborns each year, creating a market of 700 million CNY [5]. As medical reform deepens and people's health awareness increases, additional opportunities for molecular diagnostics will arise.

\subsection{Analysis of market segments of molecular diagnostics}

According to the maturity and market acceptance, molecular diagnostics can be divided into four main categories: infectious diseases, genetic diseases, oncology, and pharmacogenomics [10].

The most widely used molecular diagnostics mainly focus on the rapid and accurate diagnosis of infectious diseases, i.e., bacterial or viral infection detection. However, technological innovation and market development have broadened the application of molecular diagnostics techniques to disease susceptibility testing, early tumor diagnosis, personalized cancer therapy and prognostic evaluation, pharmacogenomics testing, birth defect detection, and forensic identification. The remarkable increase in market capacity has resulted in an average annual increase in the molecular diagnostics market of more than $30 \%$. In some emerging areas, this increase is more than $80 \%$.

Molecular diagnostics is used by individual end-users and personnel in various institutions, such as hospitals, independent medical laboratories, and physical examination centers. Molecular diagnostics techniques are divided into four categories: nucleic acid amplification technology, in situ hybridization, gene chips, and second-generation sequencing. Quantitative PCR is the most widely utilized technology, accounting for the largest 
market share. Gene chip- and microfluidic chip-based second-generation sequencing have undergone rapid development and occupy a larger market share. Although biochips were introduced in China later than in other countries, China is the first country to approve the clinical use of biochips.

\subsection{Analysis of China's biochip industry}

The global biochip market was 7.63 billion USD in 2015 and maintained a CAGR of $18.4 \%$ between 2016 and 2020, which may reach 17.75 billion USD in 2020 [11]. In 2008, the scale of China's biochip market surpassed the industrial expectation and reached 100 million USD; the estimated cost is expected to reach 900 million USD by 2020 [12]. In agreement with the overall situation of molecular diagnostics, North America is the largest market segment of biochips, while the Asia Pacific region has become the fastest-growing market segment.

China's biochip industry has been rapidly developing. The 12th Five-year Plan for Biotechnology Development promulgated by the Ministry of Science and Technology clearly states that gene chips, protein chips, and lab-on-a-chip products should be developed with independent intellectual property rights and market prospects for clinical testing and epidemic prevention. In 2011, the Ministry of Health announced that the management category of diagnostic gene chips would be adjusted from type III medical technology to type II medical technology and confirmed that its advantages included high sensitivity, high accuracy, and rapid and easy detection, making gene chips clinically applicable.

Among the enterprises that produce biochip-related products in China, Daan Gene Co., Ltd. is a listed company whose market value is 180 billion CNY. Among the unlisted companies, CapitalBio Co., Ltd. is valued at 20 billion CNY, ranking first in the entire genetic testing industry. This is followed by the Beijing Genomics Institute, with a market value of 17 billion CNY, and Berry Genomics, with a market value of 10 billion CNY [13].

Since 1998, universities, enterprises, research institutions, and some foreign agencies in China have applied for more than 2300 invention patents related to biochips. The trend in patent applications underwent a start-up period (1998-2001), wandering period (2002-2008), and rapidly increasing period (since 2009). China's biochip industry has currently attained a high level of technical competence and shows potential for continuous innovation.

Among the registration certificates of medical device products approved by the SFDA in early December 2016, 72 biochip product certificates were validated, which is considerably higher than that at the end of 2008 ( 20 certificates). As the total number of registered products increases, the number of reagent products exceeds the number of instruments, with year 2012-2013 as a watershed (Fig. 2). In the mature fields of IVD markets, such as immunological diagnosis, biochemical analysis, and other market segments, one instrument can support several reagent products. Because the number of biochip reagent products exceeds the number of instrument products, the biochip technology has entered a stage of rapid growth and mature development in the clinical diagnostics market.

Various biochip market segments are rapidly developing. With the development of two platforms (microarray chips and microfluidic chips), automatic processes are favorable for industrial transformation and clinical applications. Chips for detecting chromosomal abnormalities, screening for congenital genetic defects, and differential diagnosis of pathogens are pioneer methods in clinical testing market segments. In translational medical fields (e.g., early tumor diagnosis, prognostic evaluation, and personalized medicine), the application of biochips is increasing. Individual genetic testing is expected to extend the biochip application market to "treating before getting sick." In the field of drug discovery and development, high-content screening based on protein chips has provided support for the rapid growth of antibody drugs. Organ chips, based on microfluidic chips, have supported individualized drug discovery. The application of biochip technology has also been gradually promoted in basic

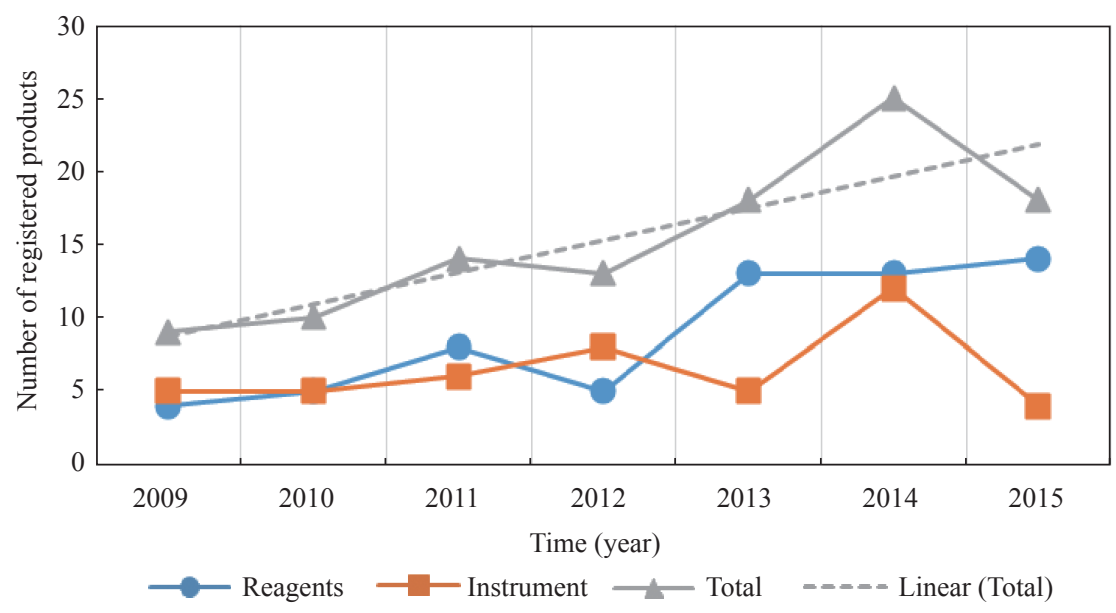

Fig. 2. Biochip-based in vitro diagnostics products approved by the SFDA from 2009 to 2015. 
industries, such as food hygiene and agricultural biotechnology, which are closely related to the national economy and people's livelihood.

\section{Challenges and problems in molecular diagnostics industry}

\subsection{China's molecular diagnostics technology has been} growing rapidly and has become competitive. Multinational corporations have increasingly favored the Chinese market, and foreign-funded enterprises gain first-mover advantages

In emerging markets, such as China, the rapid growth of the molecular diagnostics market has attracted many investors, and numerous foreign-funded enterprises have entered China. On January 17, 2012, Life Technologies and Daan Gene Co., Ltd. signed a cooperation agreement to establish an IVD technology joint venture, Life-Daan Diagnostics, to promote the IVD of genetic diseases, cancer, and infectious diseases [14]. Foreign companies, such as Roche, Bio-Rad, QIAGEN, Illumina, and PacificBio, have infiltrated and participated in the Chinese molecular diagnostics market in various manners to win first-mover advantages, which have greatly influenced the development of existing domestic molecular diagnostics enterprises. Considering that an increasing number of foreign investment are participating in the domestic molecular diagnostics market, China must perform strategic research and planning as soon as possible.

\subsection{Development of molecular diagnostics is vulnerable to regulatory constraints and is achieved through trial and error}

Regulation of the molecular diagnostics market is much stronger than that of other market segments. Most products are classified as type III products. The growth of each sub-sector is significantly affected by regulatory policies. For example, the National Health and Family Planning Commission launched a pilot program involving nucleic acid screening in blood banks in 2010. In 2014, the SFDA and National Health and Family Planning Commission urgently terminated the clinical use of gene sequencing-related products and technologies. However, the overall trend is conservative regulation and gradual liberalization, and the government exhibits a positive attitude. Therefore, leading companies in molecular diagnostics will be the largest beneficiaries in the future because of their advanced products, well-established channels, and technical reserves.

\subsection{With increasing clinical application of genetic testing, result interpretation has become a considerable challenge for medical workers}

Based on a reliable sequencing platform and optimized bioin- formatics methods, one can easily sequence large human exons and even whole genomes in labs to obtain large amounts of genetic information. However, interpreting the results and providing reasonable clinical guidance will be a great challenge for medical workers in the future.

\subsection{Policy environment should be further improved and reformed}

In China, the IVD industry must be developed in accordance with national policies. In March 2006, the National Development and Reform Commission released the "High-tech Industrialization Demonstration Project" and proposed diagnostic reagents as a special item for the first time. In October 2010, the State Council's Decision on Accelerating the Cultivation and Development of Strategic Emerging Industries was promulgated, which further emphasized the vigorous promotion of the development of diagnostic reagents and instruments [15].

The following aspect must be improved and reformed: (1) the direction of Chinese medical reform remains to be clarified, and the government bidding and purchasing system should be further improved [15]; (2) the reimbursement policy by social medical insurance and commercial medical insurance will considerably affect the innovation of molecular diagnostics products and expansion of the industrial chain. The current reimbursement policy remains limited to the routine diagnosis of diseases and does not support most molecular diagnostics innovation products limiting innovation [16].

\subsection{Industrial development experiences various challenges}

The scale of China's IVD enterprises is relatively small, and few enterprises have annual sales exceeding or close to 1 billion CNY. Further, no more than 10 enterprises have an annual sales exceeding 100 million CNY. In the future, we should focus on industrial cluster development models [14]. Awareness of intellectual property should also be improved. China has few basic patents in the upstream industry.

\subsection{Molecular diagnostics industry faces market challenges}

Industrial policy risks: In the future, China may change molecular diagnostics policies, thereby affecting the entire industry.

Influence of health insurance policy: Expansion of the molecular diagnostics market and the introduction of new products are largely influenced by the level of medical and commercial health insurance support. Current medical insurance mainly supports the treatment of a disease that has already occurred but does not favor early diagnosis and disease prediction, thereby limiting the promotion of molecular diagnostics products [16].

Risks of phasing out products or technologies: Gene chipand microfluidic chip-based sequencing are potential competi- 
tors for fluorescence quantitative PCR [16].

Risks to new product and technology development: The molecular diagnostics industry is characterized by a high risk and high output, with high requirements for the research and development $(R \& D)$ capability of enterprises. If the R\&D capability of domestic companies cannot satisfy the market demand, new products may be phased out because of their inability to compete with those from foreign companies [16].

Thresholds in the molecular diagnostics industry include specific intellectual property and non-patent technologies. However, the protection of intellectual property in China is weaker than that in foreign countries. Therefore, enterprises should accept the risk of brain drain and leakage of core technologies [16].

\subsection{Government supervision}

Overregulation of some products in China, particularly diagnostic reagent products and third-party services, may cause problems on the supply and demand sides and form a bottleneck for the industry.

\section{Strategies and suggestions}

\subsection{China should conduct a unified macro-layout for the industry and establish a national demonstration center for molecular diagnostics}

(1) Innovative development plans and R\&D strategies for molecular diagnostics should be created through a top-level design.

(2) Price approval, health insurance, and government procurement policies for innovative molecular diagnostics products and services should be developed.

\subsection{Mergers and acquisitions should be encouraged to form a complete industrial chain}

According to chain analysis of the molecular diagnostics industry, many companies provide kits, instruments, and molecular diagnostics testing services in China, whereas few companies participate in $R \& D$ and manufacturing of raw materials and high-end instruments. Companies in different industrial chains should adopt different development strategies [17].

Upstream reagent suppliers and midstream instrument suppliers can focus on product layout and enhance their own R\&D capabilities. Furthermore, channel companies can integrate channels and perform downstream services, and downstream independent diagnostic laboratories may consider increasing the proportion of special items and strengthening their cooperation with medical institutions [17]. In addition, enterprises should extend their service areas to data analysis. Currently, relatively few enterprises can provide data analysis services. Therefore, first movers will gain an advantage.

\subsection{Industrial policies related to national molecular diagnostics should be improved}

\subsubsection{Innovative leading enterprises should be cultivated}

In the case of limited resources, we should focus on the pooling of resources and supporting two to four enterprises that can compete with foreign-funded enterprises, which would benefit leading enterprises and establish an industrial model.

\subsubsection{Resource allocation should be optimized}

The role of a national demonstration center for genetic testing applications should be maximized. The development of new industrial organizations should rely on research institutes, be market-orientated, and consider enterprises as the main body. The establishment of industrial technology alliances should be supported for horizontal cooperation, support one another, and share technology and equipment. The establishment of third-party independent testing centers and other public service platforms should also be promoted. Key supports for emerging areas such as single-molecule sequencing, digital PCR, circulating tumor cell detection, and microRNA detection should also be provided.

\subsubsection{Financial policy support should be provided}

Positive and risk-free pre-, mid-, and late-investment mechanisms should be established to develop molecular diagnostics technologies and products and promote their applications. Industry-led funds should be allotted, and certain loans and interest-free policies should be offered to certain R\&D and manufacturing enterprises. IPR mortgages, shares, and other practices, which help fully embody the value of intellectual property, should be vigorously supported.

4.3.4 Market access should be provided for domestic innovative molecular diagnostics products

To address the limitations of innovative molecular diagnostics products in marketing, we should amend the bidding score sheet, weaken the market share, user feedback, product reliability, and other scoring indicators for innovative products, and directly give bonus points to innovative products. Second, the government should offer special policies, such as encouraging the use of domestic products in domestic medical institutions at all levels.

\subsubsection{Supervision should be improved}

Product review and supervision teams should be rapidly expanded and strengthened. The level of assessment and supervision should be enhanced, and the approval process of innovative products should be expedited. 


\subsection{Intellectual property strategy should be emphasized}

(1) Patent protection should be linked with foreign trade policies, and patent protection should be considered as an important component of nontariff barriers to trade negotiations.

(2) Legislation should be considered to establish patent protection in some high-tech fields that have not yet been protected by law.

\subsection{Establishment of strict and effective testing standards and strict examination and management systems should be expedited}

(1) A batch of internationally accredited laboratories or agencies should be established by states that can conduct biological evaluation of molecular diagnostics products and satisfy general safety requirements. A strict review and management mechanism should also be proposed.

(2) The training of censors and censoring experts should be strengthened to rapidly improve their ability and professionalism.

(3) Third-party clinical consulting agencies of molecular diagnostics should be founded at the national level.

\section{References}

[1] Lv J X. The application prospect of molecular diagnostics in laboratory medicine $[\mathrm{J}]$. Chinese Journal of Laboratory Medicine, 2005, 28(2): 137-139. Chinese.

[2] The worldwide in vitro diagnostic market will reach $\$ 74.7$ billion by 2020 [J]. China Pharmaceutical Technology Economics \& Management, 2014(3): 16. Chinese.

[3] Yi Y. The expansion of molecular diagnostics market has a promising prospect [N]. Medical Economic News, 2011-06-17(A02). Chinese.

[4] Wang Y. Chinese and foreign brands compete for the 20 billion in vitro diagnostics market in China [N]. China business news, 2015-
06-09. Chinese.

[5] Wang L. Project report of molecular diagnostics industry: Exploring the mystery of life, molecular diagnostic will outbreak in the future [R]. Guangzhou: Card Hang Seng Securities, 2016. Chinese.

[6] Huggett J F, Cowen S, Foy C A. Considerations for digital PCR as an accurate molecular diagnostic tool $[\mathrm{J}]$. Clinical Chemistry, 2015, 61(1): 79-88.

[7] Harris T D, Buzby P R, Babcock H, et al. Single-molecule DNA sequencing of a viral genome [J]. Science, 2008, 320(5872): 106109.

[8] Yang K, Zhang X Q, Song Y, et al. Molecular imaging techniques and applications [J]. Journal of Henan Education Institute, 2010, 19(4): 17-20. Chinese.

[9] Su X, Cheng K, Jeon J, et al. Comparison of two site-specifcally ${ }^{18} \mathrm{~F}$-labeled antibodies for PET imaging of EGFR positive tumors [J]. Molecular Pharmaceutics, 2014, 11(11): 3947-3956.

[10] Fu W L, Luo Y. Application of molecular diagnostics in nosocomial infection [J]. Chinese Journal of Nosocomiology, 2009, 19(14):1771-1775. Chinese.

[11] Wang Y. Biochip market-2016 [R]. Shanghai: MEMS Consulting, 2016. Chinese.

[12] Sohu.com. The biochip industry market is expected to reach $\$ 900$ million in 2020 [EB/OL]. (2015-06-26) [2016-11-15]. http://mt.sohu.com/20150626/n415693087.shtml. Chinese.

[13] Gene Valley. Ranking of gene detection companies [EB/OL] (2016-11-24) [2016-12-05]. http://www.genegood.com/a/ view/667.html. Chinese.

[14] Tang X L. Domestic and foreign enterprises competing admission into molecular diagnostics industry in China [N]. Medicine Economic News, 2012-11-26 (A03). Chinese.

[15] Zhang X. The complicated situation of IVD industry in China [N]. China Pharmaceutical News, 2007-11-14. Chinese.

[16] Li Y H, Zhang S Q. Retrospect and prospect of in vitro diagnostic reagent industry $[\mathrm{J}]$. Laboratory Medicine and Clinic, 2017, 14(2):299-301. Chinese.

[17] Changjiang Securities. IVD industry depth report: A rising industry [EB/OL]. (2015-07-29) [2016-11-15]. http://www.bioon.com/ trends/news/613619 2.shtml. Chinese. 